\title{
Surface waves in mesh synthetic photonic lattices
}

\author{
A.Pankov ${ }^{\mathrm{a}}$, I.Vatnik ${ }^{\mathrm{a}}$, D.Churkin ${ }^{\mathrm{a}, \mathrm{b}}$, A. A. Sukhorukov ${ }^{\mathrm{c}}$ \\ ${ }^{a}$ Novosibirsk State University, Pirogova str. 2, Novosibirsk 630090, Russia; ${ }^{\mathrm{b}}$ Aston Institute of \\ Photonic Technologies, Aston University, Birmingham, B4 7ET, United Kingdom; ${ }^{\mathrm{c} N o n l i n e a r}$ \\ Physics Centre, Research School of Physics and Engineering, The Australian National University, \\ Canberra ACT 2601, Australia
}

\begin{abstract}
Eigenmodes and dispersion curves in different configurations of synthetic photonic lattices are studied numerically. Eigenmodes localized on borders between areas with different optical potential are found. Stability of these eigenmodes against potential disturbances of different type is studied.
\end{abstract}

Keywords: synthetic photonic lattice, waveguide, surface waves, photonic topological insulator

\section{INTRODUCTION}

Controlling of light propagation is one of the central themes in the area of nanophotonics. Photonic lattices are a versatile platform for realizing and observing numerous optical phenomena. This platform can provide such properties and possibilities, which no other system can offer. One of representatives of this class is a discrete mesh lattice ${ }^{1}$. Such systems are composed of an array of waveguides with each one being discretely and periodically coupled to its adjacent neighbors (fig.1a). Unlike ordinary waveguide arrays, light propagation in such mesh systems is discretized in two dimensions (transverse and longitudinal). Pulses in such systems multiplex in transverse direction, and interfere with each other while propagating in longitudinal direction (fig. 1b). The band structure of this family of mesh lattices is derived analytically and its effects on light dynamics are investigate ${ }^{2}$. Though such systems are very complex and expensive in manufacturing, therefore another type of photonic lattices was designed - synthetic photonic lattices (SPL) (Fig1.c). These lattices are analogous to discrete mesh lattices with one important difference - signal evolution in synthetic photonic lattices takes place in time domain instead of spatial domain evolution of mesh lattices. As indicated in several studies, SPL can be used to observe a number of processes, like discrete quantum walk ${ }^{3}$, Bloch oscillations ${ }^{2}$, Anderson localization ${ }^{4,5}$, PT - symmetry ${ }^{1}$, discrete solitons ${ }^{6}$ and optical diametric drive acceleration through actionreaction symmetry breaking ${ }^{7}$. An SPL consists of two coupled fiber loops that are coupled via a central 50:50 directional coupler. These two fiber loops differ in length by $\Delta \mathrm{L}$. Here, the equivalent transverse coupling to the left and right sites is enabled by this length difference between the two loops. An independent discretization in time is then obtained by monitoring the round-trip number $m$ in these loops. Hence, the system is essentially discretized in two dimensions (fig.1d). As we will see, the propagation dynamics of light pulses, launched in such discrete temporal lattices is exactly identical to those expected in the spatial configurations. Evolution of impulse chain depends on phase of each impulse and can be altered by phase modulators. Phase modulation plays role of an optical potential in the system. Different configuration of this potential leads to different eigenmodes, that is the pulse chains with stable number of pulses within. We show analytically and numerically that controlling phase shift of light pulses by means of phase modulator it's possible to construct an analogue of a waveguide in SPL with the eigenmodes localized inside the waveguide. It is found that modes corresponding to surface waves on borders of potential wells may emerge. We investigate the evolution of surface waves in SPL, including its resistance to surface defects.

\section{SPL ANALYTICAL INVESTIGATION}

System of equations governing discrete mesh lattices can be found from general physical considerations. In this system discretized coordinate system (, $\mathrm{m}$ ) (fig.2) should be used. Intensities of signals in the left (a) and right (b) ports of the $n$th coupler are $a_{n}^{m}$ and $b_{n}^{m}$ correspondingly. It can be seen from the figure 2 that the amplitude $a_{n}^{m+1}$ is a half-sum result of the amplitudes of the pulse from the left port $\left(i a_{n-1}^{m}+b_{n-1}^{m}\right.$ with the multiplier $i$, that takes into account passing through the coupler) and from the right port $\left(a_{n}^{m}+i b_{n}^{m}\right.$ with the phase multiplier $\left.e^{i \varphi_{n}}\right)$. Amplitude of the other port

Photonic Crystal Materials and Devices XII, edited by Dario Gerace, Gabriel Lozano, Christelle Monat, Sergei G. Romanov, Proc. of SPIE Vol. 9885, 98851G · C 2016 SPIE · CCC code: 0277-786X/16/\$18 · doi: 10.1117/12.2227575 
$b_{n}^{m+1}$ is calculated in the same matter. Therefore, the system of equations describing pulses evolution in discrete mesh lattices is as follows:

$$
\left\{\begin{array}{c}
a_{n}^{m+1}=\frac{1}{2}\left[\left(a_{n}^{m}+i b_{n}^{m}\right) e^{i \varphi_{n}}+\left(-a_{n-1}^{m}+i b_{n-1}^{m}\right)\right] \\
b_{n}^{m+1}=\frac{e^{i \varphi_{n}}}{2}\left[\left(b_{n}^{m}+i a_{n}^{m}\right)+e^{i \varphi_{n+1}}\left(-b_{n+1}^{m}+i a_{n+1}^{m}\right)\right]
\end{array}\right.
$$
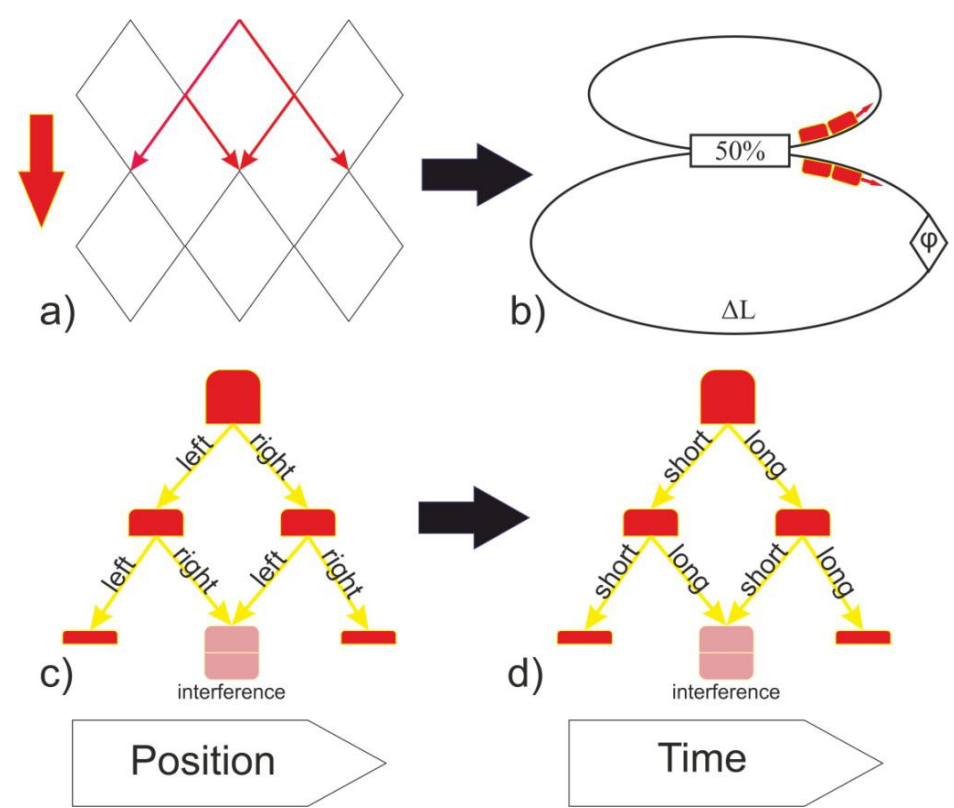

Figure 1. a) Discrete mesh lattice in the spatial domain b) Synthetic photonic lattice which is time-domain equivalent to the discrete mesh lattice c) Pulses propagation in the discrete mesh lattice in the spatial domain d) Pulses evolution in short (long) loop in time.

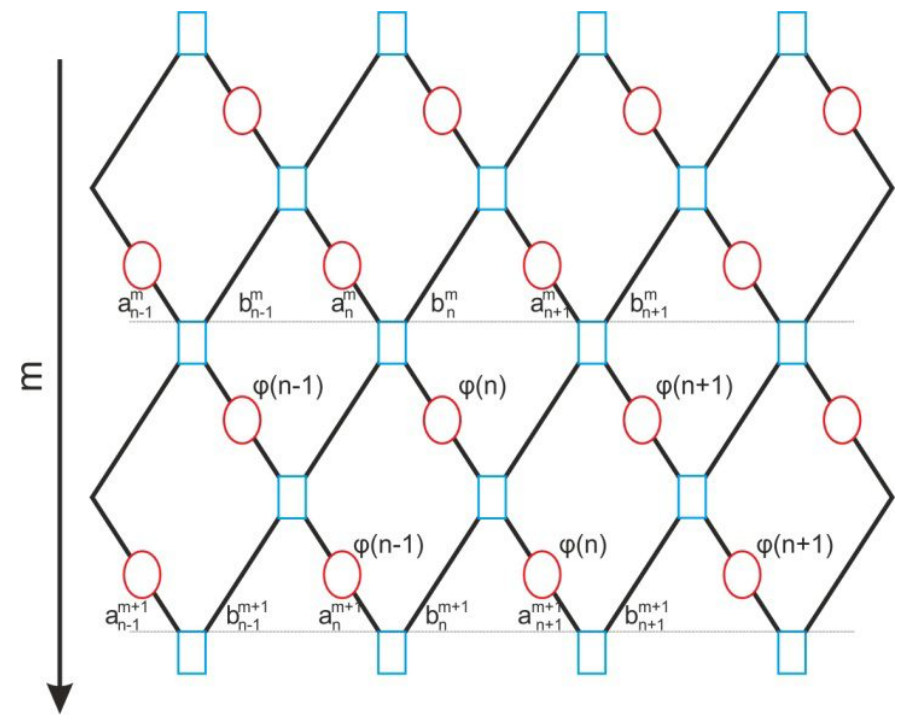

$\mathrm{n}$

Figure 2. An optical mesh lattice. The lattice is composed of an array of waveguides, which are periodically coupled together in discrete intervals. Circles indicate the position of phase elements and rectangles indicate the coupling regions. The dashed lines show the discrete points where the field intensity is evaluated before coupling occurs. 
Using such an approach it is simple to derive similar equations for a SPL:

$$
\left\{\begin{array}{c}
u_{n}^{m+1}=\frac{1}{\sqrt{2}}\left(u_{n+1}^{m}+i v_{n+1}^{m}\right) \\
v_{n}^{m+1}=\frac{e^{i \varphi_{n}}}{\sqrt{2}}\left(v_{n-1}^{m}+i u_{n-1}^{m}\right)
\end{array}\right.
$$

Where $u_{n}^{m}$ - signal intensity at the long loop during round trip $m$ and at the slot number $n, v_{n}^{m}$ - same, but at the short loop, $\varphi_{n}$ - phase shift at the slot number $n$ on the short loop.

Systems (1) and (2) are valid if the coupler coefficient is 50\%, and also these systems are equivalent [8].

In case if in synthetic photonic lattice phase is randomly distributed along the coordinate $\mathrm{n}$ and invariable along the coordinate $\mathrm{m}$, function $\varphi$ becomes independent of $\mathrm{m}$ and eigenmodes structure of such system can be found. Eigenmodes of such system can be written in the form of functions, similar to Bloch functions: $u_{n}^{m}=U_{n} \exp (\operatorname{im} \beta)$ - for a long loop, $v_{n}^{m}=V_{n} \exp (\mathrm{im} \beta)$ - for a short loop, where $\beta$ is propagation constant.

Then, considering assumption about the eigenmodes form, system of the equations for the two loops system with a random phase shift in each loop and an arbitrary coefficient of the fiber coupler can be written as follows:

$$
\left(\begin{array}{cc}
U U & U V \\
V U & V V
\end{array}\right) *\left(\begin{array}{c}
u_{1}^{m} \\
u_{2}^{m} \\
\vdots \\
u_{N}^{m} \\
v_{1}^{m} \\
v_{2}^{m} \\
\vdots \\
v_{N}^{m}
\end{array}\right)=\left(\begin{array}{c}
u_{1}^{m+1} \\
u_{2}^{m+1} \\
\vdots \\
u_{N}^{m+1} \\
v_{1}^{m+1} \\
v_{2}^{m+1} \\
\vdots \\
v_{N}^{m+1}
\end{array}\right)=\lambda *\left(\begin{array}{c}
u_{1}^{m} \\
u_{2}^{m} \\
\vdots \\
u_{N}^{m} \\
v_{1}^{m} \\
v_{2}^{m} \\
\vdots \\
v_{N}^{m}
\end{array}\right)
$$

, where matrices UU, UV, VU, VV - are defined as follows:

$$
\begin{aligned}
& U U=\left(\begin{array}{ccccc}
0 & 0 & 0 & \ldots & \cos (\theta) \exp \left(i \varphi_{1}\right) \\
\cos (\theta) \exp \left(i \varphi_{2}\right) & 0 & \ldots & \ldots & 0 \\
0 & \cos (\theta) \exp \left(i \varphi_{3}\right) & 0 & \cdots & 0 \\
\vdots & \vdots & \ddots & \cos (\theta) \exp \left(i \varphi_{N}\right) & 0 \\
0 & \cdots & 0 & &
\end{array}\right) \\
& U V=\left(\begin{array}{ccccc}
0 & 0 & 0 & \ldots & i \sin (\theta) \exp \left(i \varphi_{1}\right) \\
i \sin (\theta) \exp \left(i \varphi_{2}\right) & 0 & \ldots & \ldots & 0 \\
0 & i \sin (\theta) \exp \left(i \varphi_{3}\right) & 0 & \ddots & 0 \\
\vdots & \vdots & \ddots & i \sin (\theta) \exp \left(i \varphi_{N}\right) & 0 \\
0 & \cdots & 0 & &
\end{array}\right) \\
& V U=\left(\begin{array}{ccccc}
0 & i \sin (\theta) \exp \left(i \varphi_{1}\right) & 0 & \cdots & 0 \\
0 & 0 & i \sin (\theta) \exp \left(i \varphi_{2}\right) & \cdots & \vdots \\
\vdots & \vdots & 0 & \ddots & 0 \\
0 & \vdots & \vdots & \ddots & i \sin (\theta) \exp \left(i \varphi_{N-1}\right) \\
i \sin (\theta) \exp \left(i \varphi_{N}\right) & 0 & \cdots & 0 & 0
\end{array}\right) \\
& V V=\left(\begin{array}{ccccc}
0 & \cos (\theta) \exp \left(i \varphi_{1}\right) & 0 & \cdots & 0 \\
0 & 0 & \cos (\theta) \exp \left(i \varphi_{2}\right) & \cdots & \vdots \\
\vdots & \vdots & 0 & \ddots & 0 \\
0 & \vdots & \vdots & \ddots & \cos (\theta) \exp \left(i \varphi_{N-1}\right) \\
\cos (\theta) \exp \left(i \varphi_{N}\right) & 0 & \cdots & 0 & 0
\end{array}\right)
\end{aligned}
$$

, where $\theta$ depends on fiber coupler coefficient ( $\pi / 4$ for $50 \%$ ). 
Solving matrix equation (3) eigenvalues and eigenvectors of this system can be found. Eigenvalue and corresponding eigenvector form an eigenmode, and propagation constants $\beta_{j}=\log \left(\lambda_{j}\right) / i$ versus modenumber $\mathrm{j}$ form a dispersion curve. To present system (1) as a matrix equation (3) it was necessary to enter the boundary conditions. They are provided by terms $(1, \mathrm{~N})$ in matrices $\mathrm{UU}$ and $\mathrm{VV}$, and by terms $(\mathrm{N}, 1)$ in matrices $\mathrm{VU}$ and $\mathrm{VV}$.

If two phase modulators are installed on both loops and their phase shifts are constant $\left(\varphi_{n}^{m}=\varphi, \psi_{n}^{m}=\psi\right)$ then it is possible to derivate an analytical formula for propagation constant $\beta$. In this case, solutions of the matrix equation (3) can be formulated as:

$$
u_{n}^{m}=U \mathrm{e}^{i K n+i m \beta}, \quad v_{n}^{m}=\mathrm{Ve}^{i K n+i m \beta}
$$

where $\mathrm{K}$ is a transverse wavenumber. Substituting eq. (4) into the eq. (3) we obtain an eigenmode equation:

$$
\left(\begin{array}{cc}
e^{i(\varphi-K) \cos (\theta)} & i e^{i(\varphi-K) \sin (\theta)} \\
i e^{i(\psi+K) \sin (\theta)} & e^{i(\psi+K) \cos (\theta)}
\end{array}\right)\left(\begin{array}{l}
U \\
V
\end{array}\right)=e^{i \beta}\left(\begin{array}{l}
U \\
V
\end{array}\right)
$$

Its solution is found as:

$$
e^{\beta_{ \pm}}=e^{i\left(\frac{\varphi+\psi}{2}\right)\left[\cos (K+\psi-\varphi) \cos (\theta) \pm \sqrt[2]{\cos ^{2}(K+\psi-\varphi) \cos ^{2}(\theta)-1}\right]}
$$

and finally:

$$
\beta_{ \pm}=\frac{\varphi+\psi}{2} \pm \cos ^{-1}[\cos (K+\psi-\varphi) \cos (\theta)]
$$

This formula is responsible for the shape of the dispersion curve.

Given arbitrary $\varphi_{n}^{m}$ and $\psi_{n}^{m}$ dependences, one can calculate the eigenvectors and eigenvalues using the approach above.

\section{SPL WAVEGUIDE}

In SPL, with the help of phase modulators, it is possible to create a time domain analogue of waveguide structure (fig. 3 b). Different phase shifts in the different areas (fig.3a) allow us to put a propagation constants of a one area into the bandgaps of other areas (fig.4), thus creating a waveguide effect. Signals propagations and corresponding dispersion curves were calculated for this system configuration with different values of phase shift $\varphi$. At the curtain value of phase shift $(\varphi=\pi)$ set of eigenmodes contains modes that correspond to propagation only inside the waveguide area and modes corresponding to propagation only outside of that area (fig.5).

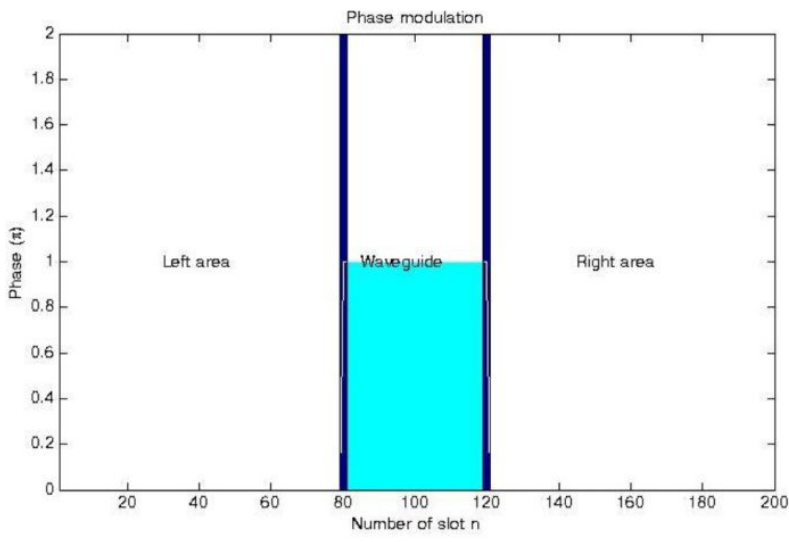

a)

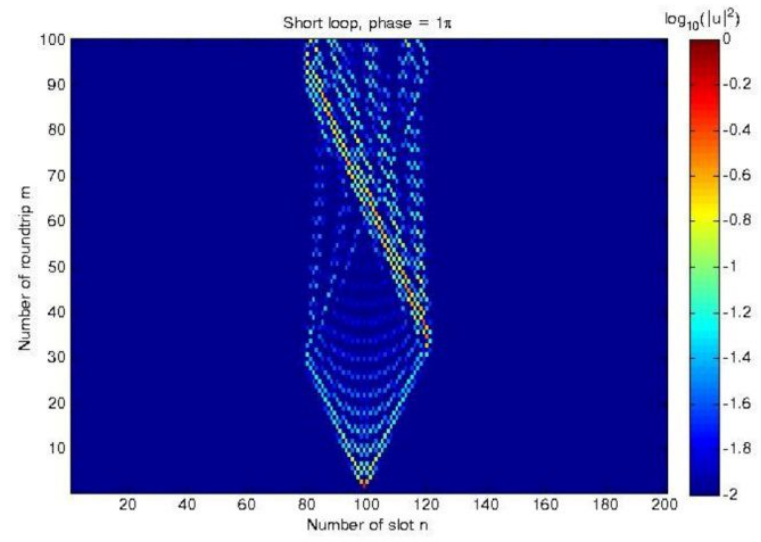

b)

Figure 3. a) Phase modulation dependence on slot number $n$ b) Corresponding signal evolution on the short loop (waveguide). 


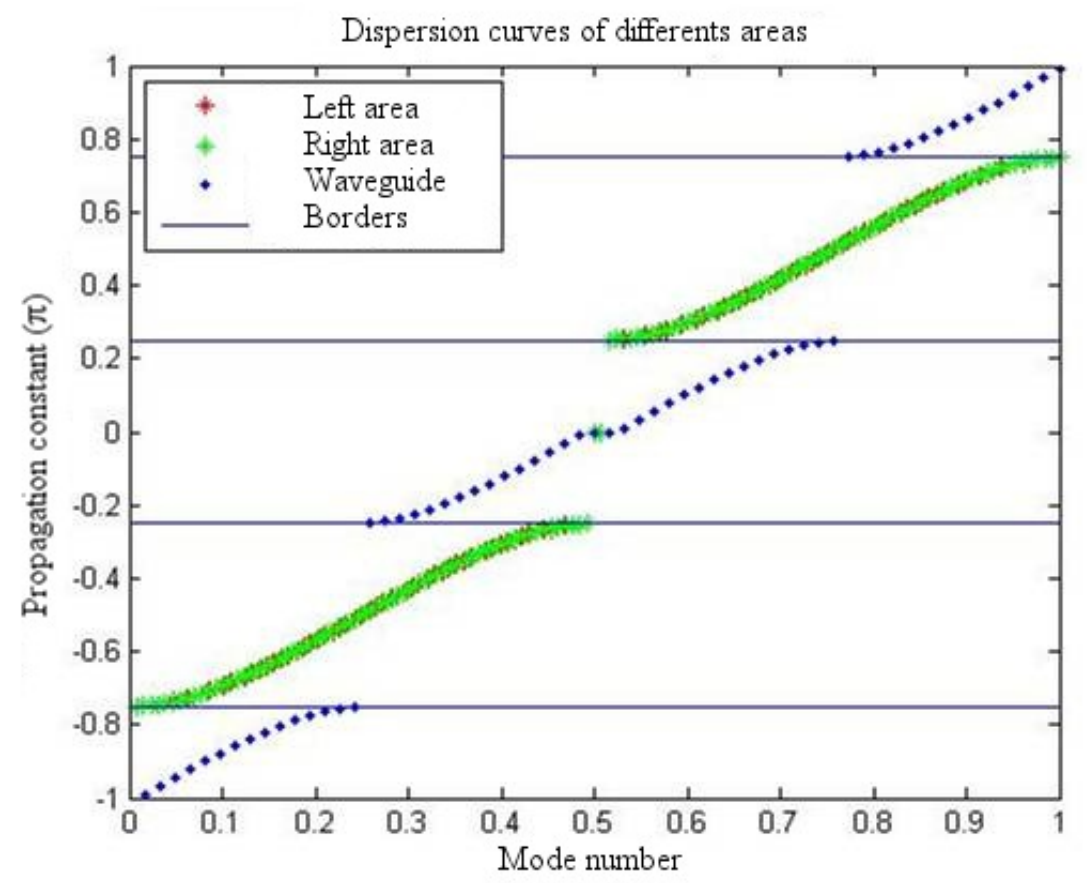

Figure 4. Dispersion curves of waveguide and areas on the left and on the right doesn't overlap
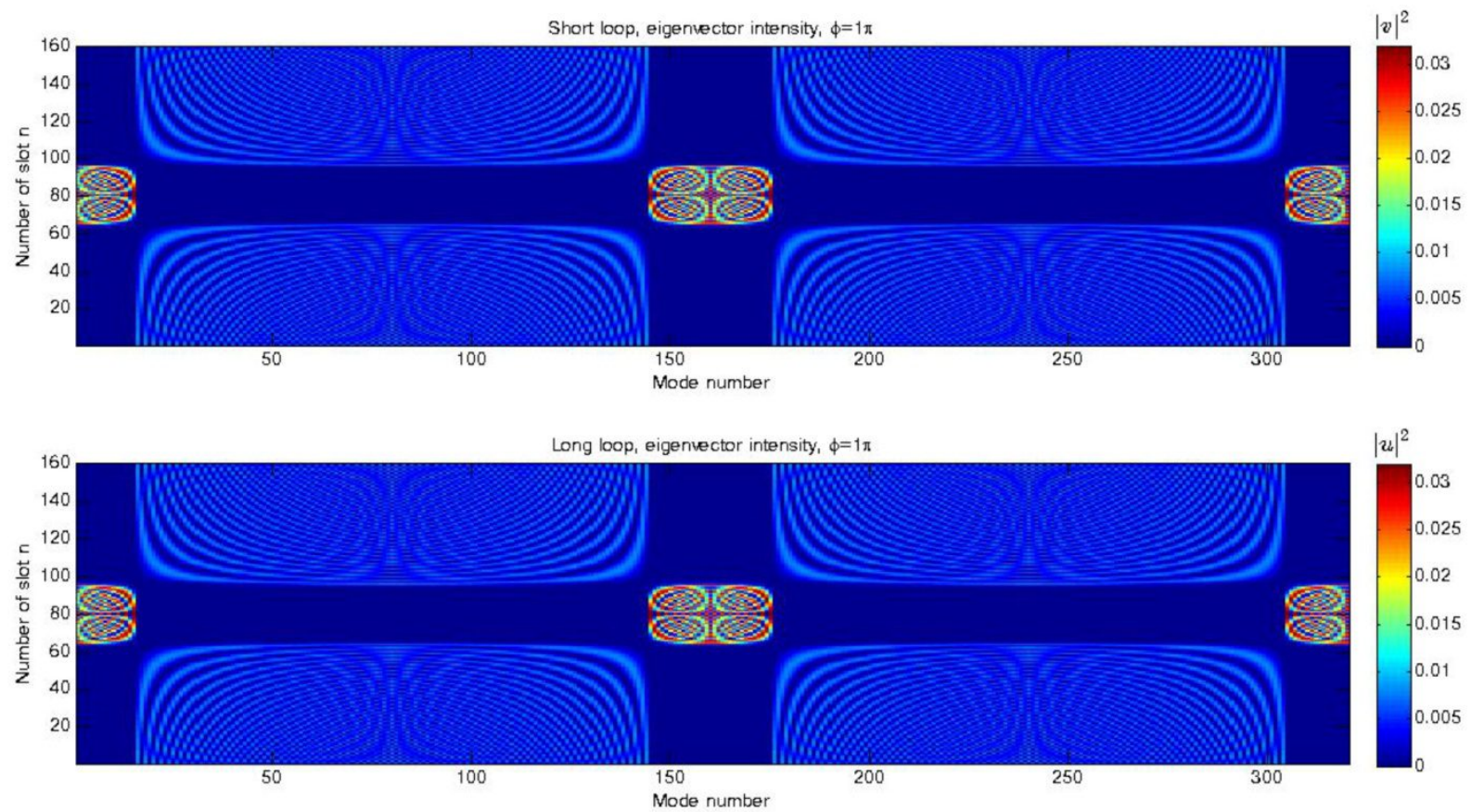

Figure 5. Eigenvectors, corresponding to waveguide effect in SPL, of the short and the long loops.

\section{SURFACE WAVES}

Different configurations of SPL waveguide were studied. When using two phase modulators, on a short loop and long loop respectively, which works in antiphase regime $(\varphi=-\psi)$, formula for propagation constant takes the following form: 


$$
\beta_{ \pm}= \pm \cos ^{-1}[\cos (K-2 \varphi) \cos (\theta)]
$$

This formula shows that it is possible to shift dispersion curves of the different areas in SPL along the K axis only. This is impossible in traditional waveguide arrays. At specific values of phase shift $\varphi$ dispersion curves of waveguide area (we still call area with the phase shift a waveguide area, though there is no waveguide effect at this configuration) and outside areas are completely overlap, and though all their values lies in the same intervals, surface waves, located on the boundaries between areas only, may appear. This effect was never demonstrated in SPL. Results of modulation are demonstrated on the figures 6-8. It is clear from the figure 6 that there are four modes containing surface waves and their eigenvalues lie inside the bandgap. Corresponding eigenvectors on the figure 7 shows that every mode corresponds to a surface wave only on one border.

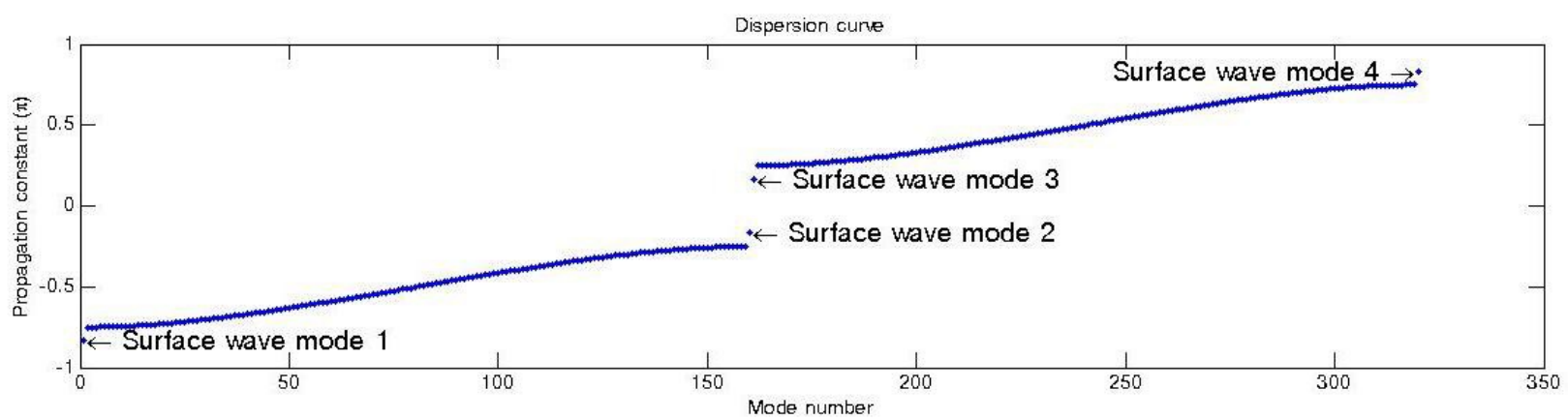

Figure 6. Dispersion curve of all SPL, not divided on areas.
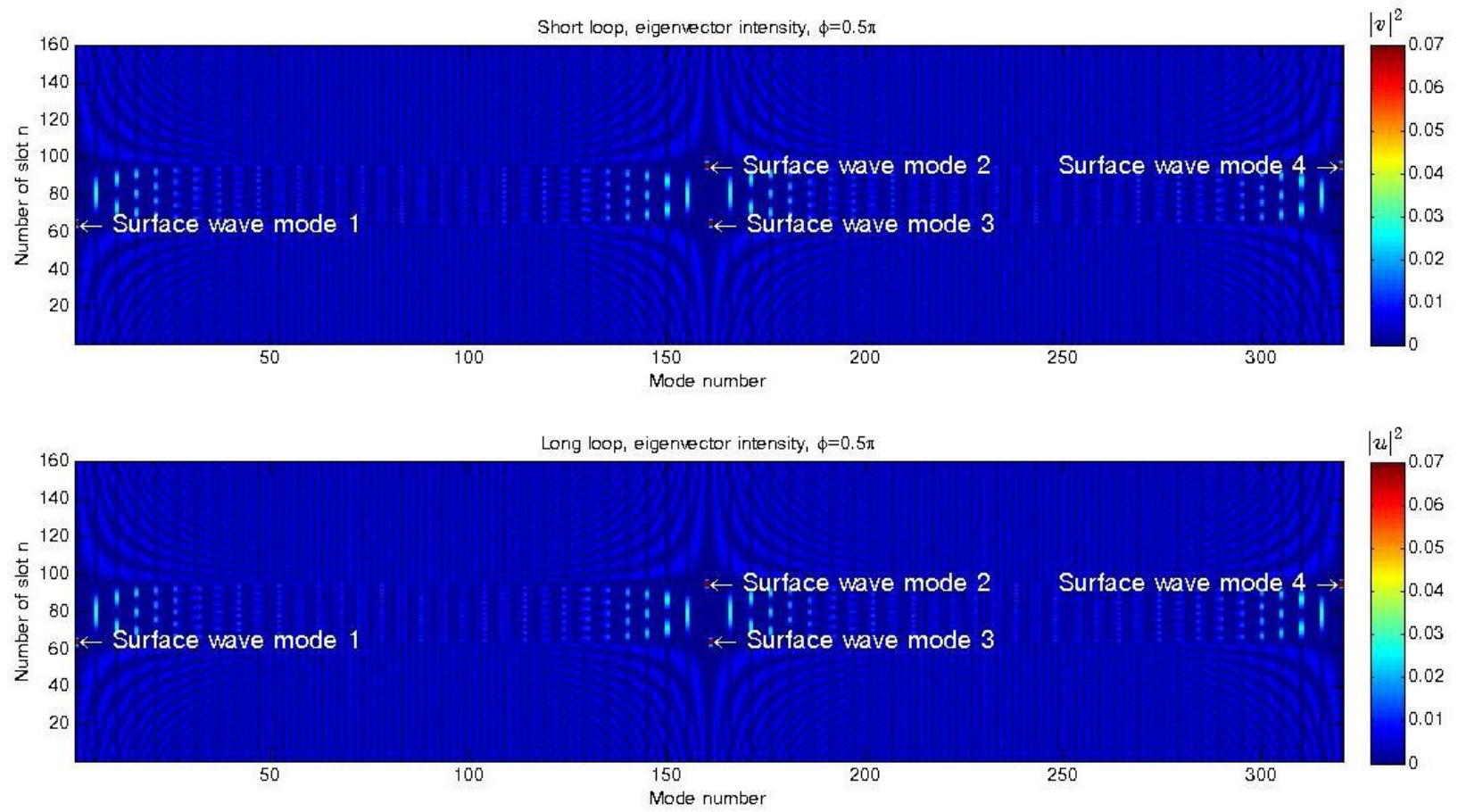

Figure 7. Eigenvectors, corresponding to surface waves in SPL, of the short and the long loops. 

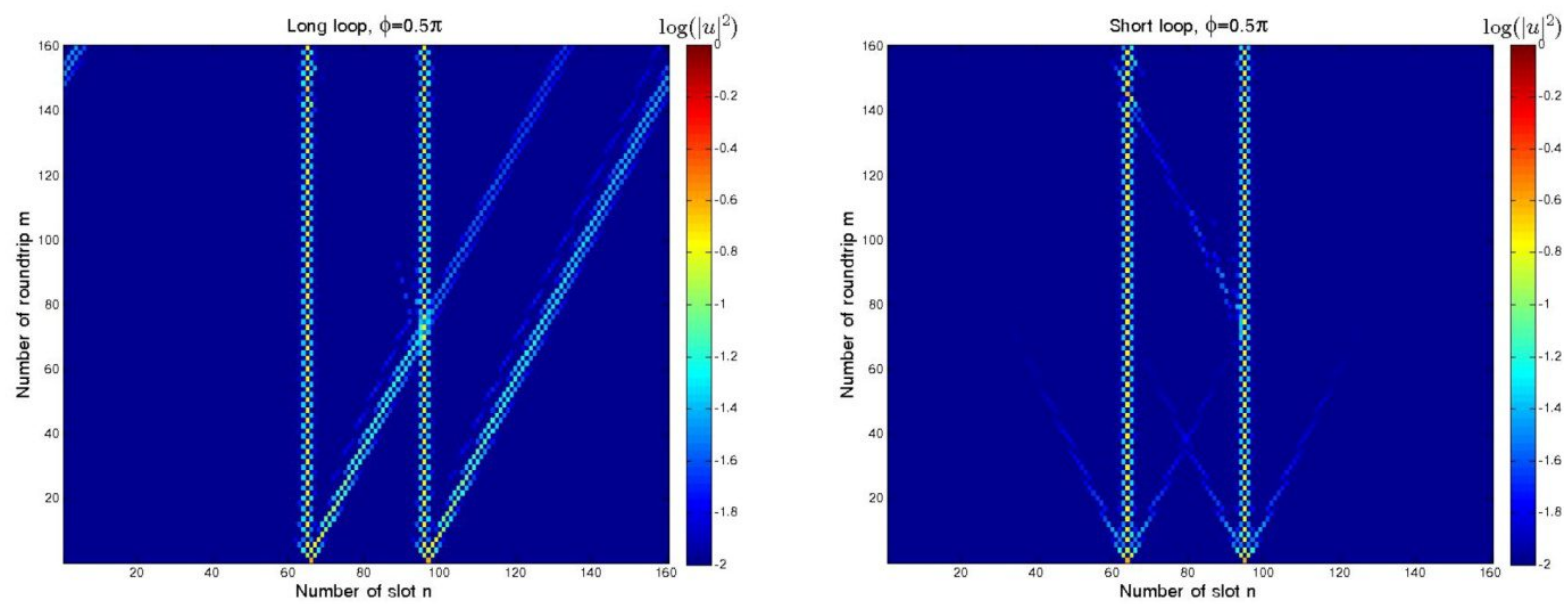

Figure 8. Signal propagation on the short and the long loops (situated in waveguide borders).

\section{WAVES STABILITY}

Surface waves resistance to different kinds of waveguide surface defects was studied in order to find out if such SPL configurations have properties of photonic topological insulators.

Topological insulator is a material, which behaves as an insulator in its interior, but whose surface conducts electricity. In two-dimensional case electrons on the surface of the topological insulator aren't scattered by defects situated at the surface. Numerous scientific works are directed on realization of electro-magnetic waves topological insulator, including experimentally demonstrated two-dimensional Floquete topological insulator [9] based on composition of an array of evanescently coupled helical waveguides arranged in a graphene-like honeycomb lattice. Other group reported imaging of topological edge states in silicon photonics $[10,11]$.

Resistance of surface waves in SPL to point defects of different sizes was studied. On a simple point defect partial energy dissipation happens (fig.9d) Varying transverse size of the defect ( $\mathrm{n}$ axes) doesn't change the result, amount of dissipated energy and energy in the form of a surface wave stay the same. Increase of the defect size along the longitudinal direction ( $\mathrm{m}$ axes) increase the amount of dissipated energy, until, at the size of the defect $\Delta \mathrm{m}=7$, surface waves completely destroyed. Sizes of the point defect demonstrated on the figure 9c. Study of resistance of surface waves to other types of defects, like randomly distributed surface defects (fig.9a) or different periodical surface structure (fig.9b) showed the same effect of destruction of surface waves.
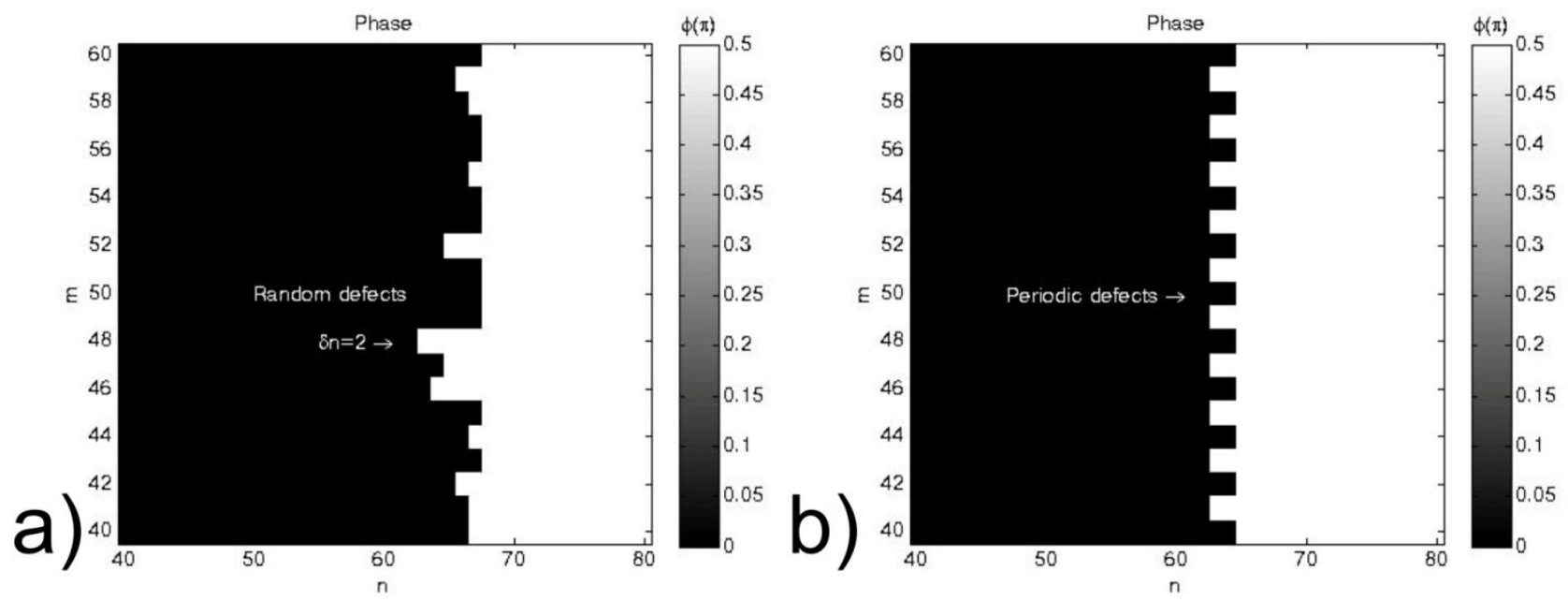

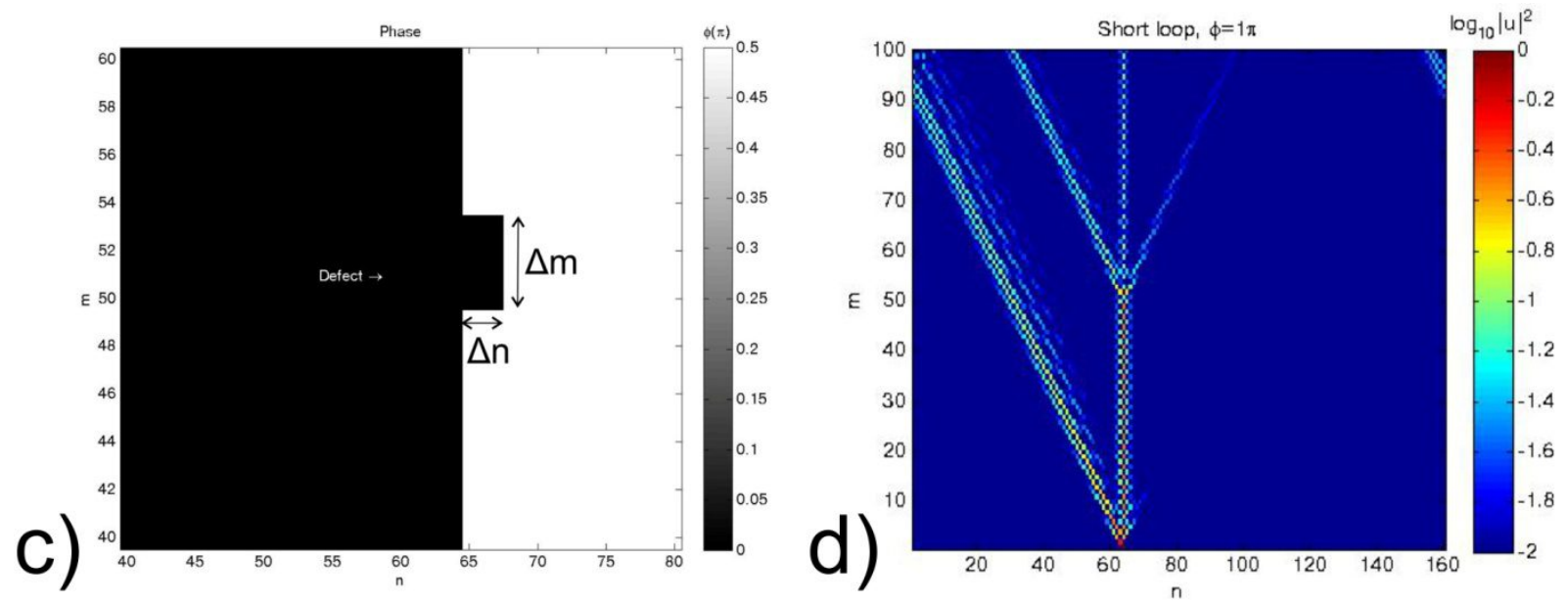

Figure 9. a) Randomly distributed surface defects b) Periodical surface defect c) Point defect d) Signal evolution in the short loop showing dissipation of a surface mode on a point surface defect.

\section{CONCLUSION}

We have demonstrated an existence of surface waves in synthetic photonic lattices, which are localized at the borders of regions the different optical potentials. In order to create surface waves, dispersion curves in different areas should be shifted along the propagation constant axis, that is easily achieve in synthetic photonic lattices. The surface waves turned out not to be stable against surface defects.

\section{REFERENCES}

[1] Miri, M. A., Regensburger, A., Peschel, U., \& Christodoulides, D. N. "Optical mesh lattices with PT symmetry" Physical Review A 86(2), 023807 (2014).

[2] Schreiber, A., Cassemiro, K. N., Potoček, V., Gábris, A., Mosley, P. J., Andersson, E. Silberhorn, C. ”Photons walking the line: a quantum walk with adjustable coin operations" Physical review letters 104(5), 050502 (2010)

[3] Schreiber, A., Cassemiro, K. N., Potoček, V., Gábris, A., Jex, I., Silberhorn, C. "Decoherence and disorder in quantum walks: from ballistic spread to localization" Physical review letters 106(18), 180403 (2011).

[4] Regensburger, A., Bersch, C., Hinrichs, B., Onishchukov, G., Schreiber, A., Silberhorn, C., Peschel, U. "Photon propagation in a discrete fiber network: An interplay of coherence and losses" Physical review letters 107(23), $233902(2011)$.

[5] Vatnik, I. D., Tikan, A. M., Churkin, D. V., \& Sukhorukov, A. A. "Anderson Localization In Optical Mesh Lattices Realized In Time Domain” In The European Conference on Lasers and Electro-Optics (p. CD_P_8) (2015)

[6] Wimmer, M., Regensburger, A., Miri, M. A., Bersch, C., Christodoulides, D. N., Peschel, U. "Observation of optical solitons in PT-symmetric lattices" Nature communications 6 (2015).

[7] Wimmer, M., Regensburger, A., Bersch, C., Miri, M. A., Batz, S., Onishchukov, G. Peschel, U. “Optical diametric drive acceleration through action-reaction symmetry breaking" Nature Physics 9(12), 780-784 (2013).

[8] Regensburger, A., Miri, M. A., Bersch, C., Näger, J., Onishchukov, G., Christodoulides, D. N., Peschel, U. "Observation of Defect States in P T-Symmetric Optical Lattices" Physical review letters 110(22), 223902 (2013).

[9] Rechtsman, M. C., Zeuner, J. M., Plotnik, Y., Lumer, Y., Podolsky, D., Dreisow, F. Szameit, A. "Photonic Floquet topological insulators" Nature 496(7444), 196-200 (2013).

[10] Hafezi, M., Mittal, S., Fan, J., Migdall, A., \& Taylor, J. M. "Imaging topological edge states in silicon photonics" Nature Photonics 7(12), 1001-1005 (2013)

[11] Mittal, S., Fan, J., Faez, S., Migdall, A., Taylor, J. M., \& Hafezi, M. (2014). Topologically robust transport of photons in a synthetic gauge field. Physical review letters, 113(8), 087403. 